\title{
Convective instabilities in two superposed horizontal liquid layers heated laterally
}

\author{
S. Madruga, ${ }^{1,2, *}$ C. Pérez-García, ${ }^{2,3}$ and G. Lebon ${ }^{1}$ \\ ${ }^{1}$ Departement AGO, Liège University, B5, Sart-Tilman, B-4000 Liège, Belgium \\ ${ }^{2}$ Instituto de Física, Universidad de Navarra, E-31080 Pamplona, Navarra, Spain \\ ${ }^{3}$ Departamento de Ingeniería Mecánica, E. T. S. Ingenieros (TECNUN), Universidad de Navarra, E-20018 San Sebastián, Spain
}

(Received 13 February 2003; published 23 October 2003)

\begin{abstract}
This work is devoted to the theoretical study of the stability of two superposed horizontal liquid layers bounded by two solid planes and subjected to a horizontal temperature gradient. The liquids are supposed to be immiscible with a nondeformable interface. The forces acting on the system are buoyancy and interfacial tension. Four different flow patterns and temperature profiles are found for the basic state. A linear perturbative analysis with respect to two- and three-dimensional perturbations reveals the existence of three kinds of patterns. Depending on the relative height of both liquids several situations are predicted: either wave propagation from cold to the hot regions, or waves propagating in the opposite direction or still stationary longitudinal rolls. The behavior of three different pairs of liquids which have been used in experiments on bilayers under vertical gradient by other authors have been examined. The instability mechanisms are discussed and a qualitative interpretation of the different behaviors exhibited by the system is provided. In some configurations it is possible to find a codimension-two point created by the interaction of two Hopf modes with different frequencies and wave numbers. These results suggest to consider two liquid layers as an interesting prototype for the study of propagation and interaction of waves in the context of the Bénard-Marangoni problem.
\end{abstract}

DOI: 10.1103/PhysRevE.68.041607

PACS number(s): 47.20.Dr, 47.20.Bp, 47.54.+r, 47.27.Te

\section{INTRODUCTION}

There has been recently an increasing interest in the study of buoyant-thermocapillary flows induced by horizontal thermal gradients [1]. Such situations occur in oceanographic currents due to nonhomogeneous heating, and their interplay with the atmosphere gives rise to complex meteorological phenomena. They are also important in crystal growth. The floating zone process generates thermocapillary convective cells in the region of crystallization, which require better understanding and control.

When a horizontal temperature gradient is acting on a horizontal liquid layer enclosed between two horizontal walls, the problem is much more complicated than in the more classical case of vertical heating. First, the reference state is no longer at rest, giving rise to a general flow and a nonlinear vertical temperature profile. A nontrivial problem will therefore consist in determining the basic reference temperature and velocity fields. Another peculiarity is that the threshold of instability depends on the Prandtl number Pr. The problem of thermocapillary instabilities induced by a lateral heating was first studied by Smith and Davis [2]. They predicted the presence of hydrothermal waves and stationary rolls. More details are provided in a subsequent work by Smith [3], who showed the existence of two different mechanisms of instability depending on Pr. At low Pr, the energy necessary to sustain the disturbances comes from the horizontal applied temperature field and the hydrothermal waves propagate in a direction perpendicular to the horizontal temperature gradient. At high Pr, the energy is extracted from the vertical temperature field by vertical convection and it gives rise to hydrothermal waves propagating parallel to the tem-

*Electronic address: smadruga@fisica.unav.es perature gradient. At intermediate Pr, the mechanism is a combination of the previous effects and leads to waves forming an angle with the streamwise direction. Parmentier et al. [4] studied the coupling of buoyant and thermocapillarydriven instabilities, in the case of systems with lower and upper insulating bounding surfaces within a the threedimensional (3D) linear formulation. Their numerical results display the three kinds of behaviors described above. For thermally conducting surfaces, Gershuni et al. [5] find stationary rolls for $\operatorname{Pr}>1$. Other theoretical works have mainly focused on comparison with experiments. Mercier and Normand [6] performed a linear analysis to explain the experimental results by Daviaud and Vince [7] for a silicon oil with $\operatorname{Pr}=10$. They notice that the transition between traveling waves and stationary rolls observed when the liquid depth is increased depends on the heat transfer between the liquid and its environment. In their discussion about absolute, global, and convective instabilities Priede and Gerbeth [8] show that the threshold of absolute instability fits better the experimental data of $1 \mathrm{cS}$ silicon oil $(\operatorname{Pr}=13.9)$ [9] than the value predicted from convective instability. A nonlinear analysis of stability for purely thermocapillary convection has been carried out by Smith [10], who determines the range of existence of the two oblique traveling waves predicted by the linear theory. More recently, new results have been reported [11] on hydrothermal waves in different geometries, measuring their frequency, wave number, and angle of propagation.

In the previous theoretical works a liquid layer surmounted by a gas phase that remains passive (one-layer approximation) was considered. In some cases $[6,8]$ the effect of the adjacent gas is modeled by a Biot number, a phenomenological parameter that characterizes the heat transfer between liquid and gas. The Biot number was originally defined for steady conditions; however, when convection takes place, the dynamics of thermal and mechanical perturbations 
in the gas may have a strong influence on the convection inside the liquid. In such a case, the introduction of a Biot number becomes questionable and the one-layer approximation should be revised. In the general case, a full two-layer hydrodynamical description is required. Furthermore, wellcontrolled experiments involve always two fluid layers [12]. Such systems are typical of many practical situations such as Earth's mantle convection or encapsulated crystal growth.

In the past, most theoretical [13] and experimental studies $[12,14-16]$ about Rayleigh-Bénard convection in two immiscible liquids have been devoted to vertical heating. Only few contributions are found dealing with a horizontal temperature gradient. To our knowledge the first work on the subject is by Villers and Platten [17]; they measured the velocity profiles in each layer as a function of the height in a system formed by water and heptanol. They also developed a simple theoretical model to calculate the horizontal velocity profile as a function of the ratio of viscosities, expansion coefficients, and thicknesses of both layers. Later on, Doi and Koster [18] studied theoretically the thermocapillary convection under microgravity conditions in two immiscible liquid layers with a free upper surface, in 2D geometries. They determine under which conditions the lower layer remains at rest. Moreover, they carry out numerical simulations in a box of aspect ratio 4 in order to analyze the effect of the vertical walls. Numerical simulations in cavities of different aspect ratios for coupled thermocapillary and buoyancy-driven convection were performed by Liu et al. [19], and an asymptotic solution for the velocity in the limit of infinite aspect ratio has also been derived [20].

The objective of the present work is to study coupled thermocapillary and buoyancy convection in a two-layer system of infinite horizontal extension subject to lateral heating and to find out the regimes occurring in this configuration. A linear approach to the problem is carried out, showing the kind of oscillatory and stationary behaviors that occur in this system.

The paper is organized as follows: In Sec. II, the general equations governing the problem are established. Section III is devoted to the derivation of the temperature and velocity profiles of the basic flow, the explicit expressions are reported in the Appendix. In Sec. IV is carried out a linear stability analysis of the basic state towards bidimensional and three-dimensional perturbations. Conclusions are drawn in Sec. VI.

\section{PROBLEM FORMULATION}

We consider a system of two horizontal superimposed immiscible liquid layers of thickness $h^{(i)}$, densities $\rho^{(i)}$, kinematic viscosities $\nu^{(i)}$, coefficients of volume expansion $\left(\alpha^{(i)}\right)$, thermal conductivities $\lambda^{(i)}$, and thermal diffusivities $\kappa^{(i)}$, superscript $i=1,2$ refers to the lower and upper fluid respectively. The system is infinite in the horizontal extension and is limited in the vertical direction by two horizontal rigid and thermally conductive walls (see Fig. 1). The interface between both liquids is assumed to be horizontal and nondeformable. The origin of the Cartesian reference system is fixed at the interface.

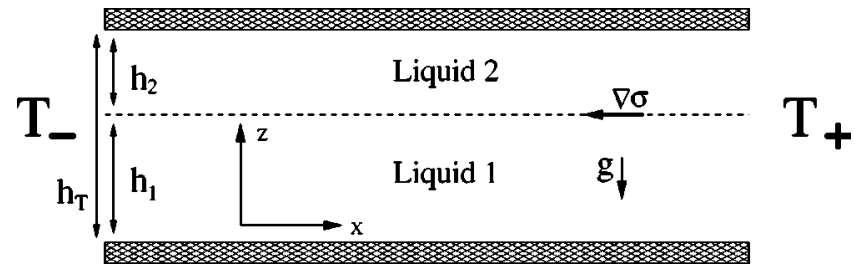

FIG. 1. Diagram of the two-layer system under study. Two superposed immiscible liquids bounded horizontally by conductive rigid walls are subject to a horizontal gradient of temperature. The interface is supposed to be nondeformable. The gravity and interfacial tension are the forces acting on the system.

The system is heated, with a constant temperature gradient $\beta$ imposed along the horizontal direction, which produces a conducting temperature profile given by $T=T_{-}$ $+\beta x$, where $T_{-}$is the temperature of the cold side. The Boussinesq approximation is taken for granted in both layers. The system is subject to the gravity field and its density is given by the state equation $\rho^{(i)}=\rho_{0}^{(i)}\left[1-\alpha^{(i)}\left(T-T_{-}\right)\right]$. The interfacial tension which acts at the interface admits a temperature variation approximated by the linear equation of state $\sigma=\sigma_{0}-\gamma\left(T-T_{-}\right)$where $\gamma=-\partial \sigma / \partial T$ is a positive constant (at least for the pairs of liquids considered in this work).

The governing equations expressing the balance of mass, momentum, and energy in the Boussinesq approximation are given by

$$
\begin{gathered}
\boldsymbol{\nabla} \cdot \mathbf{u}^{(i)}=0 \\
\frac{\partial \mathbf{u}^{(i)}}{\partial t}+\left(\mathbf{u}^{(i)} \cdot \boldsymbol{\nabla}\right) \mathbf{u}^{(i)}=-\frac{\boldsymbol{\nabla} p^{(i)}}{\rho_{0}^{(i)}}-g\left[1-\alpha\left(T^{(i)}-T_{-}\right)\right] \hat{\mathbf{z}} \\
+\nu^{(i)} \nabla^{2} \mathbf{u}^{(i)} \\
\frac{\partial T^{(i)}}{\partial t}+\left(\mathbf{u}^{(i)} \cdot \boldsymbol{\nabla}\right) T^{(i)}=\kappa^{(i)} \nabla^{2} T^{(i)}
\end{gathered}
$$

wherein $\boldsymbol{u}^{(i)}=\left(u^{(i)}, v^{(i)}, w^{(i)}\right)$ is the velocity field, $p^{(i)}$ the pressure, $g$ the acceleration of the gravity, and $\hat{\mathbf{z}}$ the unit vector in the vertical direction. The boundary conditions at the rigid and thermally conductive bottom and upper walls are

$$
\begin{gathered}
z=-h^{(1)} \rightarrow \mathbf{u}^{(1)}=\mathbf{0} ; \quad T^{(1)}=T_{w}\left(T_{w}=T_{-}+\beta x\right), \\
z=h^{(2)} \rightarrow \mathbf{u}^{(2)}=0 ; \quad T^{(2)}=T_{w}\left(T_{w}=T_{-}+\beta x\right) .
\end{gathered}
$$

Furthermore, one must include the conditions of continuity of the temperature, heat flow, and velocity at the interface (the normal velocity component is zero because the interface is supposed to be nondeformable),

$$
\begin{gathered}
z=0 \rightarrow \mathbf{u}_{h}^{(1)}=\mathbf{u}_{h}^{(2)}, \quad w^{(1)}=w^{(2)}=0, \quad T^{(1)}=T^{(2),} \\
\lambda^{(1)} \partial_{z} T^{(1)}=\lambda^{(2)} \partial_{z} T^{(2)}, \quad \partial_{z} \mathbf{u}_{h}^{(2)}-\partial_{z} \mathbf{u}_{h}^{(1)}=-\frac{d \sigma}{d T} \nabla_{h} T^{(1)},
\end{gathered}
$$


the last relation in Eq. (3) expresses the balance between the tangential stresses at the interface. To transform the governing equations and boundary conditions in dimensionless form, the following scales are selected: for length $h^{(1)}$, time $h^{(1) 2} / \kappa^{(1)}$, velocity $\kappa^{(1)} / h^{(1)}$, pressure $\rho^{(1)} \nu^{(1)} \kappa^{(1)} / h^{(1) 2}$, and temperature $\beta h^{(1)}$. In terms of nondimensional quantities, the balance Eqs. (1) for the lower liquid read as

$$
\begin{gathered}
\boldsymbol{\nabla} \cdot \mathbf{u}^{(1)}=0, \\
\operatorname{Pr}^{-1}\left[\frac{\partial \mathbf{u}^{(1)}}{\partial t}+\left(\mathbf{u}^{(1)} \cdot \boldsymbol{\nabla}\right) \mathbf{u}^{(1)}\right] \\
=-\nabla p^{(1)}-\left[\frac{\left(h^{(1)}\right)^{3} g}{\nu^{(1)} \kappa^{(1)}}-\operatorname{Ra}\left(T^{(1)}-T_{-}\right)\right] \hat{\mathbf{z}}+\nabla^{2} \mathbf{u}^{(1)},
\end{gathered}
$$

$$
\frac{\partial T^{(1)}}{\partial t}+\left(\mathbf{u}^{(1)} \cdot \boldsymbol{\nabla}\right) T^{(1)}=\nabla^{2} T^{(1)},
$$

while for the upper liquid

$$
\begin{gathered}
\boldsymbol{\nabla} \cdot \mathbf{u}^{(2)}=0 \\
\operatorname{Pr}^{-1}\left[\frac{\partial \mathbf{u}^{(2)}}{\partial t}+\left(\mathbf{u}^{(2)} \cdot \boldsymbol{\nabla}\right) \mathbf{u}^{(2)}\right] \\
=-\frac{1}{\rho} \boldsymbol{\nabla} p^{(2)}-\left[\frac{\left(h^{(1)}\right)^{3} g}{\nu^{(1)} \kappa^{(1)}}-\alpha \operatorname{Ra}\left(T^{(2)}-T_{-}\right)\right] \hat{\mathbf{z}} \\
+\nu \nabla^{2} \mathbf{u}^{(2)}, \\
\quad \frac{\partial T^{(2)}}{\partial t}+\left(\mathbf{u}^{(2)} \cdot \nabla\right) T^{(2)}=\kappa \nabla^{2} T^{(2)}
\end{gathered}
$$

in Eqs. (4) and (5) and eliminating the pressure one obtains

The nondimensional boundary conditions are

$$
\begin{gathered}
z=-1 \rightarrow \mathbf{u}^{(1)}=\mathbf{0}, \quad T^{(1)}=T_{w}\left(T_{w}=T_{-}+x\right) ; \\
z=a \rightarrow \mathbf{u}^{(2)}=\mathbf{0}, \quad T^{(2)}=T_{w}\left(T_{w}=T_{-}+x\right) ; \\
z=0 \rightarrow \mathbf{u}^{(1)}=\mathbf{u}^{(2)}, \quad w^{(1)}=w^{(2)}=0, \quad T^{(1)}=T^{(2)} ; \\
\partial_{z} T^{(1)}=\lambda \partial_{z} T^{(2)}, \quad \mu \partial_{z} \mathbf{u}_{h}^{(2)}-\partial_{z} \mathbf{u}_{h}^{(1)}=\mathrm{Ma}_{h} T^{(1)} .
\end{gathered}
$$

In Eqs. (4)-(6) the following nondimensional parameters have been introduced: $\alpha=\alpha^{(2)} / \alpha^{(1)}, \quad \kappa=\kappa^{(2)} / \kappa^{(1)}, \quad \nu$ $=\nu^{(2)} / \nu^{(1)}, \lambda=\lambda^{(2)} / \lambda^{(1)}, a=h^{(2)} / h^{(1)}, \rho=\rho^{(2)} / \rho^{(1)}$, and $\mu=\rho^{(2)} \nu^{(2)} / \rho^{(1)} \nu^{(1)}=\rho \nu$. The Prandtl number is defined with respect to the liquid 1, i.e., $\operatorname{Pr}=\nu^{(1)} / \kappa^{(1)}$, as well as the Rayleigh number $\mathrm{Ra}=\alpha^{(1)} \beta g\left(h^{(1)}\right)^{4} / \nu^{(1)} \kappa^{(1)}$ and the Marangoni number $\mathrm{Ma}=-(d \sigma / d T)\left[\beta\left(h^{(1)}\right)^{2} / \rho^{(1)} \nu^{(1)} \kappa^{(1)}\right]$.

\section{THE BASIC STATE}

When a horizontal gradient is imposed, a stationary basic flow sets in each liquid [2] with a horizontal velocity component depending on the vertical coordinate $\mathbf{u}^{(i)}$ $=\left[u_{0}^{(i)}(z), 0,0\right]$. On the other hand, the basic temperature the following equations governing the behavior of the velocity and temperature fields in both liquids:

$$
\begin{gathered}
\partial_{z^{3}} u_{0}^{(1)}=\mathrm{Ra}, \quad \partial_{z^{2}} \tau^{(1)}=u_{0}^{(1)} ; \\
\nu \partial_{z^{3}} u_{0}^{(2)}=\alpha \mathrm{Ra}, \quad \kappa \partial_{z^{2}} \tau^{(2)}=u_{0}^{(2)},
\end{gathered}
$$

with the corresponding boundary conditions

$$
\begin{gathered}
z=-1 \rightarrow u_{0}^{(1)}=0, \quad \tau^{(1)}=0 ; \\
z=a \rightarrow u_{0}^{(2)}=0, \quad \tau^{(2)}=0 ; \\
z=0 \rightarrow \tau^{(1)}=\tau^{(2)}, \quad u_{0}^{(1)}=u_{0}^{(2)} ; \\
\partial_{z} \tau^{(1)}=\lambda \partial_{z} \tau^{(2)}, \quad \mu \partial_{z} u_{0}^{(2)}-\partial_{z} u_{0}^{(1)}=\mathrm{Ma} .
\end{gathered}
$$

profile is the superposition of the imposed horizontal gradient and the vertical profile $\tau^{(i)}(z)$ generated by the fluid motion: $T^{(i)}=T_{-}+x+\tau^{(i)}(z)$ (in nondimensional form). To calculate $u_{0}^{(i)}(z)$, and $\tau^{(i)}(z)$, we replace

The return flow condition [2] which requires that the net flow through a vertical plane must vanish in each layer, i.e.,

$$
\int_{-1}^{0} u_{0}^{(1)}=0, \quad \int_{0}^{a} u_{0}^{(2)}=0
$$

provides two additional conditions that allow us to calculate explicitly the velocity and temperature fields $u_{0}^{(i)}$ and $\tau^{(i)}$, whose expressions in terms of $z$ are found in the Appendix.

As shown in Eqs. (A1)-(A4) the basic velocity and temperature profiles are the sum of a thermocapillary term proportional to the Marangoni number Ma and a buoyancy term proportional to the Rayleigh number $\mathrm{Ra}$. The velocity profile is a second-order polynomial in $z$ in the interfacial term and of third order in the buoyancy, whereas the temperature profile is fourth order in the interfacial term and fifth in the buoyancy.

The velocity and temperature profiles depend on the ratios of the various transport coefficients and depths. For a given experimental setup the heating does not affect the shape of the basic velocity and temperature profiles since the temperature gradient appears as a constant factor in $u_{0}^{(i)}$ and $\tau^{(i)}$ through the Rayleigh and Marangoni numbers.

Each velocity profile has a root located at its corresponding rigid wall [as imposed by the boundary conditions (6a) and (6b)]. There is also a second root inside each layer due to the return flow condition. In absence of gravity, this root is located at $z=-1 / 3$ in the lower layer and at $z=a / 3$ in the upper fluid, i.e., one-third of the depth counted from the interface, and the flow consists of one convective cell in each layer. When gravity is taken into account, the positions of these roots depend on the properties of the fluids and a third root can be present, allowing for a second convective cell in each layer. The temperature profiles also have a root located 


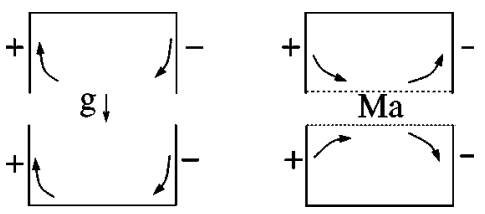

FIG. 2. Driving forces: gravity (left) and interfacial tension (right). The buoyancy and thermocapillary forces act in the same direction in the lower layer, and in opposite direction in the upper layer where they are competing.

at the bounding horizontal walls. In presence of gravity it is possible to find up to five roots in each layer.

\section{Velocity and temperature profiles}

The flow is driven by interfacial tension gradients, and by density differences when the gravity is acting. As usually, it is assumed that the surface tension decreases with temperature $(\gamma>0)$, so that a horizontal temperature gradient gives rise to tangential forces that drive the fluid from hot to cold regions. Therefore, with only thermocapillary effects present, a general circulation around the interface is established, which will drag the fluid from the hot to the cold side. In a finite container, by continuity, the fluid in the upper layer rises near the cold side and falls down near the hot one; on the contrary, in the lower layer it falls along the cold side and raises up at the hot one. One would therefore observe two counterrotating cells. Buoyancy forces also drive the fluid from hot (lower density) to cold regions (higher density). Since the fluid rises along the hot side and falls at the colder one, in both cells, the buoyancy will favor the formation of two corotating cells. In coupled thermocapillary-buoyancy convection, buoyancy and interfacial forces are acting in the same direction in the lower layer, just like in one-liquid systems with $\gamma>0$. However, they act in opposite directions in the upper layer, where the forces are competing (see Fig. 2). Thus the liquid 2 exhibits a scenario similar to that of a one-liquid system with $\gamma<0$. (This occurs in some ceramics and liquid alloys such as $\mathrm{Ag} \mathrm{Pb},[21]$.)

In Figs. 3(a) and 3(b) are displayed the basic velocity and temperature profiles. Along the horizontal axis is reported the total depth $h_{T}$ of the two superposed fluids while the vertical axis gives the percentage of depth of the lower liquid with respect to the total depth, i.e., the fractional bottom depth $\hat{h}_{1}$ $\left[\hat{h}_{1}=100 /\left(1+h^{(2)} / h^{(1)}\right)\right]$. The curves refer to a configuration formed by perfluorinated HT-70 (Galden Heat Transfer fluid HT 70) (lower fluid) and $5 \mathrm{cS}$ silicon oil (upper fluid), this pair of fluids has been used in a recent experiment with vertical heating by Juel et al. [15]. (The physical properties of these liquids are listed in Table I.) In both figures, four different regions denoted by (I) - (IV) are distinguished. Let us pay attention to Fig. 3(a). The region (I) extends to all $h_{T}$ and covers principally high values of $\hat{h}_{1}$. The basic state consists of a clockwise convective cell in liquid 2, and a counterclockwise cell in liquid 1; in this region the direction of the general circulation is governed by the interfacial forces. In region (II), the competition between buoyancy and interfacial forces gives birth to two sublayers in liquid 2. The motion in the sublayer close to the interface is dominated by the interfacial forces, but near the top wall it is the buoyancy that contributes to create a second sublayer. In regions (I) and (II), the general flow structure in liquid 1 is not changed by the motion of the upper liquid. However, for high $h_{T}$ and small $\hat{h}_{1}$ the motion in layer 1 is driven by the upper liquid. This is the case of regions (III) and (IV), where the direction of circulation close to the interface changes its sign. This is so because the buoyancy force acting on liquid 2 is so strong that it does not only drive the motion in the upper layer but it also modifies the flow direction near the interface. In (III) two convection cells are found in liquid 1 , but the one close to the lower surface is no longer driven by liquid 2 . In region (IV) one observes the scenario opposite to (I), i.e., two convection cells but with the liquid flowing from the cold to the hot side near the interface. (The behavior of the lower liquid
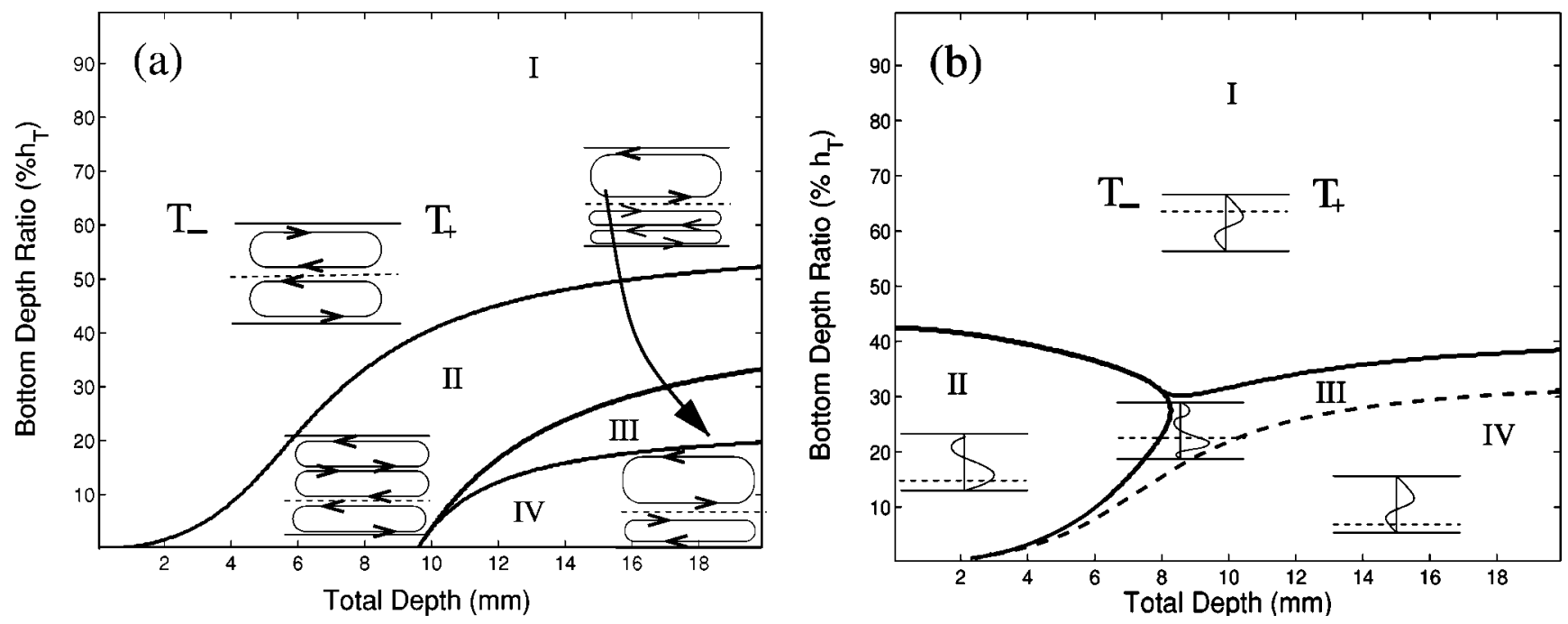

FIG. 3. Velocity profiles (a) and temperature profiles (b) of the basic state for a system composed by perfluorinated HT-70 (lower liquid) and $5 \mathrm{cS}$ silicon oil (upper liquid). The dashed line gives the position of $M 1$ (see the text). 
TABLE I. Parameter values of the three pairs of liquids studied. The parameter ratio in each different configuration is also given. The Crispation number $\mathrm{Cr}$ is given for a depth of $h^{(1)}=3 \mathrm{~mm}$.

\begin{tabular}{|c|c|c|c|c|c|c|c|c|}
\hline Liquid & $\begin{array}{c}\rho \\
\left(\mathrm{kg} / \mathrm{m}^{3}\right)\end{array}$ & $\left(10^{-6} \mathrm{~m}^{2} / \mathrm{s}\right)$ & $\begin{array}{c}\lambda \\
(\mathrm{J} / \mathrm{m} \mathrm{s} \mathrm{K})\end{array}$ & $\begin{array}{c}c_{p} \\
(\mathrm{~J} / \mathrm{kg} \mathrm{K})\end{array}$ & $\begin{array}{c}\alpha \\
\left(10^{-3} \mathrm{~K}^{-1}\right)\end{array}$ & $\begin{array}{c}\gamma \\
(\mathrm{N} / \mathrm{m} \mathrm{K})\end{array}$ & $\operatorname{Pr}$ & $\mathrm{Cr}$ \\
\hline Silicon oil 5 cS & 920 & 5 & 0.117 & 1590 & 1.05 & & 62.512 & \\
\hline HT-70 & 1680 & 0.5 & 0.07 & 962 & 1.1 & & 11.54 & \\
\hline Silicon oil 5 cS/HT-70 & 0.548 & 10 & 1.671 & 1.653 & 0.954 & $-7.3 \times 10^{-5}$ & & $2.1 \times 10^{-6}$ \\
\hline Water & 997 & 0.893 & 0.609 & 4180 & 0.257 & & 6.111 & \\
\hline Fc-75 & 1760 & 0.945 & 0.063 & 1046 & 1.4 & & 27.397 & \\
\hline Water/Fc-75 & 0.566 & 0.945 & 9.59 & 3.996 & 0.183 & $-4.7 \times 10^{-5}$ & & $3.3 \times 10^{-7}$ \\
\hline$n$-Hexane & 655 & 0.458 & 0.12 & 2270 & 1.41 & & 5.675 & \\
\hline Acetonitrile & 776 & 0.476 & 0.118 & 2230 & 1.41 & & 4.381 & \\
\hline$n$-Hexane/Acetonitrile & 0.844 & 0.962 & 1.017 & 1.018 & 1 & $-1 \times 10^{-4}$ & & $7.8 \times 10^{-7}$ \\
\hline
\end{tabular}

is analogous to that of a single liquid with $\gamma<0$ [21].) Contrary to the zero-gravity problem [18], states with one of the two layers at rest are not found in the present problem.

Let us now examine the temperature field in the basic state [Fig. 3(b)]. The four regions do not coincide with these velocity fields but they are related. Region (I) covers more than half the surface of Fig. 3(b). Liquid 2 is unstably stratified (temperature increases with depth), in liquid 1 one finds a lower layer close to the wall which is unstably stratified, and above it one distinguishes a stably stratified sublayer (temperature decreases with depth). Region (II) is the only closed area: the lower liquid 1 is stably stratified whereas the upper liquid 2 is characterized by two stable stratified sublayers adjacent to the wall and the interface, in the middle there is an unstable stratified region, giving rise to a S-shaped profile. Region (III) shows the most complicated temperature profile: liquid 2 exhibits two unstably stratified sublayers at the boundaries and a stable sublayer in the intermediate region, liquid 1 consists in three sublayers, the intermediate being stably stratified while the two others are unstably stratified. Zone (IV) covers the low $\hat{h}_{1}$ and almost all $h_{T}$ values, the global profile is similar to that of region (I), with the interface located at smaller $\hat{h}_{1}$ so that liquid 1 is unstably stratified.

\section{EVOLUTION EQUATIONS FOR THE PERTURBATIONS}

As soon as a horizontal temperature difference $\Delta T$ is applied, convective cells typical of the basic state set in, but a further increase of $\Delta T$ may destabilize this basic flow. To analyze the stability of the reference state with respect to infinitesimally small perturbations, let us write the general solution of the problem under the form $\mathbf{u}^{(i)}=\left(u_{0}^{(i)}\right.$ $\left.+u^{\prime(i)}, v^{\prime(i)}, w^{\prime(i)}\right), \quad T^{(i)}-T_{-}=x+\tau^{(i)}+\theta^{\prime(i)}, \quad p^{(i)}=p_{0}^{(i)}$ $+p^{\prime(i)}$, where $\left(u^{\prime(i)}, v^{\prime(i)}, w^{\prime(i)}\right), \theta^{\prime(i)}$ and $p^{\prime(i)}$ denote the perturbations of velocity, temperature, and pressure fields, respectively.

The perturbations are decomposed into a sum of normal modes $\quad\left(\mathbf{u}^{\prime}, \theta^{\prime}, p^{\prime}\right)=\left[\mathbf{u}^{\prime}(z), \theta^{\prime}(z), p^{\prime}(z)\right] \operatorname{expi}(\mathbf{k} \cdot \mathbf{x}+\omega t)$, where $\omega$ denotes a complex frequency and $\mathbf{k}$ the wave number, with component $k_{x}$ in the streamwise direction and $k_{y}$ in the spanwise direction. The primes will be dropped for clarity in the ensuing equations. After eliminating the pressure in the momentum equation and omitting nonlinear terms, we are left with the following equations for the perturbations, for the lower liquid 1 ,

$$
\begin{gathered}
\partial_{z^{2}} u^{(1)}=L^{(1)} u^{(1)}+\frac{k_{y}^{2}}{k^{2}} \operatorname{Pr}^{-1} \partial_{z} u_{0}^{(1)} w^{(1)} \\
+i \frac{k_{x}}{k^{2}}\left(\partial_{z^{3}} w^{(1)}-L^{(1)} \partial_{z} w^{(1)}\right) \\
\partial_{z^{4} w^{(1)}=}\left(L^{(1)}+k^{2}\right) \partial_{z^{2}} w^{(1)}-L^{(1)} k^{2} w^{(1)}+\operatorname{Ra}^{2} \theta^{(1)} \\
-i k_{x} \operatorname{Pr}^{-1} w^{(1)} \partial_{z^{2}} u_{0}^{(1)}, \\
\partial_{z^{2}} \theta^{(1)}=\left[k^{2}+i\left(\omega+u_{0}^{(1)} k_{x}\right)\right] \theta^{(1)}+u^{(1)}+\partial_{z} \tau^{(1)} w^{(1)},
\end{gathered}
$$

for the upper liquid 2,

$$
\begin{aligned}
& \nu \partial_{z^{2}} u^{(2)}=L^{(2)} u^{(2)}+\frac{k_{y}^{2}}{k^{2}} \operatorname{Pr}^{-1} \partial_{z} u_{0}^{(2)} w^{(2)} \\
& +i \frac{k_{x}}{k^{2}}\left(\nu \partial_{z^{3}} w^{(2)}-L^{(2)} \partial_{z} w^{(2)}\right), \\
& \nu \partial_{z^{4 w}} w^{(2)}=\left(\nu k^{2}+L^{(2)}\right) \partial_{z^{2} w^{(2)}}-k^{2} L^{(2)} w^{(2)}+\operatorname{Ra} k^{2} \alpha \theta^{(2)} \\
& -i k_{x} \operatorname{Pr}^{-1} w^{(2)} \partial_{z^{2}} u_{0}^{(2)}, \\
& \kappa \partial_{z^{2}} \theta^{(2)}=\left[\kappa k^{2}+i\left(\omega+u_{0}^{(2)} k_{x}\right)\right] \theta^{(2)}+u^{(2)}+\partial_{z} \tau^{(2)} w^{(2)} \text {, }
\end{aligned}
$$

wherein the following notation has been used:

$$
\begin{gathered}
L^{(1)}=k^{2}+i \operatorname{Pr}^{-1}\left(\omega+u_{0}^{(1)} k_{x}\right), \\
L^{(2)}=\nu k^{2}+i \operatorname{Pr}^{-1}\left(\omega+u_{0}^{(2)} k_{x}\right) .
\end{gathered}
$$

The corresponding boundary conditions are

$$
\begin{gathered}
z=-1 \rightarrow u^{(1)}=w^{(1)}=\partial_{z} w^{(1)}=\theta^{(1)}=0 ; \\
z=a \rightarrow u^{(2)}=w^{(2)}=\partial_{z} w^{(2)}=\theta^{(2)}=0 ; \\
z=0 \rightarrow u^{(1)}=u^{(2)}, \quad \partial_{z} w^{(1)}=\partial_{z} w^{(2)}, \quad w^{(1)}=w^{(2)}=0,
\end{gathered}
$$



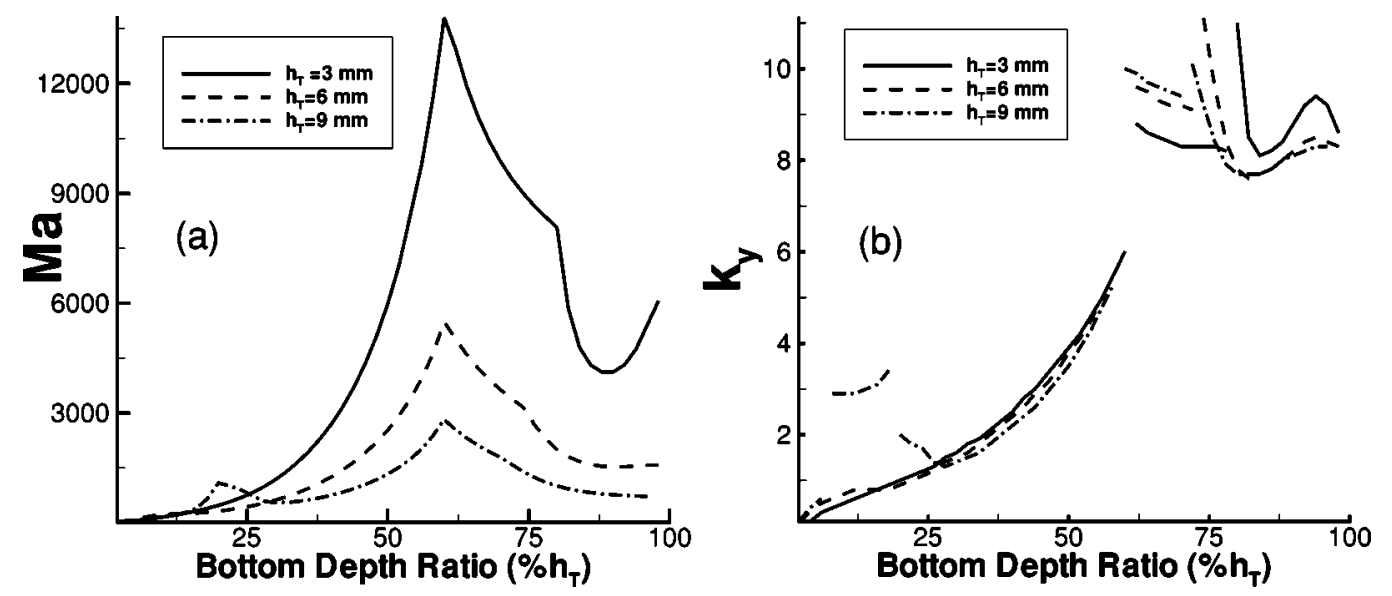

FIG. 4. Critical Marangoni (left) and critical wave number (right) for 2D longitudinal perturbations $\left(k_{x}=0\right)$ as a function of the bottom depth ratio, for three different values of the total depth. The results correspond to the pair HT-70 (lower liquid) and $5 \mathrm{cS}$ silicon oil (upper liquid).

$$
\begin{gathered}
\theta^{(1)}=\theta^{(2)}, \quad \partial_{z} \theta^{(1)}=\lambda \partial_{z} \theta^{(2)}, \\
\mu \partial_{z} u^{(2)}-\partial_{z} u^{(1)}=i k_{x} \operatorname{Ma} \theta^{(1)}, \\
\mu \partial_{z}^{2} w^{(2)}-\partial_{z}^{2} w^{(1)}=\operatorname{Ma} k^{2} \theta^{(1)} .
\end{gathered}
$$

We are faced with an eigenvalue problem which is solved by means of a Tau-Chebyshev spectral method, by approximating the eigenfunctions with Chebyshev polynomials of order 16. The marginal curves are found by searching the set of values $k_{x}, k_{y}$, Ma for which the rate of temporal growth is zero.

\section{RESULTS OF THE LINEAR ANALYSIS}

Three mechanisms are able to destabilize the system under study. Basic temperature profiles in Fig. 3(b) present unstably stratified zones (temperature increases with depth) and other zones which are stably stratified (temperature decreases with depth), in the unstably stratified regions the RayleighBénard instability may arise. A second mechanism is the usual Bénard-Marangoni instability when the interface is colder than the adjacent fluid. Finally, a third destabilizing mechanism arises even when the core of the layers is colder than the interface, provided the velocity flow is strong enough to overcome the stabilizing vertical temperature field [3].

Owing to the great number of parameters involved in a two-layer configuration, the results will be discussed in the case of selected pairs of liquids used in experiments. The calculations have been made for the pair $5 \mathrm{cS}$ silicon oil (upper liquid) and perfluorinated HT-70 (lower liquid), but later on we also consider the combinations: water (upper fluid) with perfluorinated hydrocarbon Fc-75 (3M Fluorinert FC-75) (lower liquid), and $n$-hexane (upper liquid) with acetonitrile (lower liquid). The parameter values of these fluids are gathered in Table I. Deformability is generally quantified by means of the so-called crispation number, defined as $\mathrm{Cr}$ $=\rho^{(1)} \nu^{(1)} \kappa^{(1)} / \sigma_{0} h^{(1)}$. As shown in Table I the values of $\mathrm{Cr}$ are very small for the pairs of liquids examined in the present work. Then the hypothesis of nondeformability of the interface is reasonable as it has been shown in earlier publications $[22,23]$. Moreover, we will not pay attention to the particular $\hat{h}_{1}$ values close to $0 \%$ or $100 \%$ because in these cases thinfilm motions (outside the scope of the present analysis) would appear.

\section{A. Stationary longitudinal rolls}

First, we consider 2D perturbations characterized by $k_{x}$ $=0$ and $\omega=0$ (longitudinal stationary rolls). Our analysis extends to two-layer systems the studies by Gershuni et al. [5], for a single-fluid layer bounded by horizontal rigid-free and perfectly thermal conductive surfaces, and by Mercier and Normand [6], who considered the heat transfer at the free surface by means of a Biot number.

Figure 4 displays the critical Marangoni and the critical spanwise wave number versus the fractional bottom depth for several values of the total depth $h_{T}(3,6$, and $9 \mathrm{~mm})$. For total depths of 3 and $6 \mathrm{~mm}$, one observes three stationary branches, while for $h_{T}=9 \mathrm{~mm}$ an additional branch is clearly seen for low $\hat{h}_{1}$. Figure 4(a) shows that the critical Marangoni number decreases with $h_{T}$. This is due to the destabilizing effect of the increase of depth in RayleighBénard instability. All the curves exhibit the most stable zone for $\hat{h}_{1} \approx 60 \%$. The jump from one branch to another is better appreciated by the discontinuities in $k_{y}$ in Fig. 4(b). The spanwise wave number $k_{y}$ for $h_{T}=3 \mathrm{~mm}$ and $h_{T}=6 \mathrm{~mm}$ increases with $\hat{h}_{1}$ in the first branch, however, it decreases in the second one together with that of $h_{T}=9 \mathrm{~mm}$, the dependence with $\hat{h}_{1}$ in the third branch is more involved for the three depths. In the branch jump the marginal Marangoni curve is bimodal [6], switching the absolute minimum between two wave numbers. This modal change gives rise to different states in the two-layer system.

In Fig. 5 are represented the isotherms and the velocity fields in the $z-y$ plane as observed at the critical threshold. At small fractional bottom depths $\left[\hat{h}_{1}=14 \%\right.$ in Fig. $\left.5(\mathrm{a})\right]$ strong temperature gradients are found in the bulk of liquid 1, whereas in liquid 2 they are concentrated near the interface 
(a)
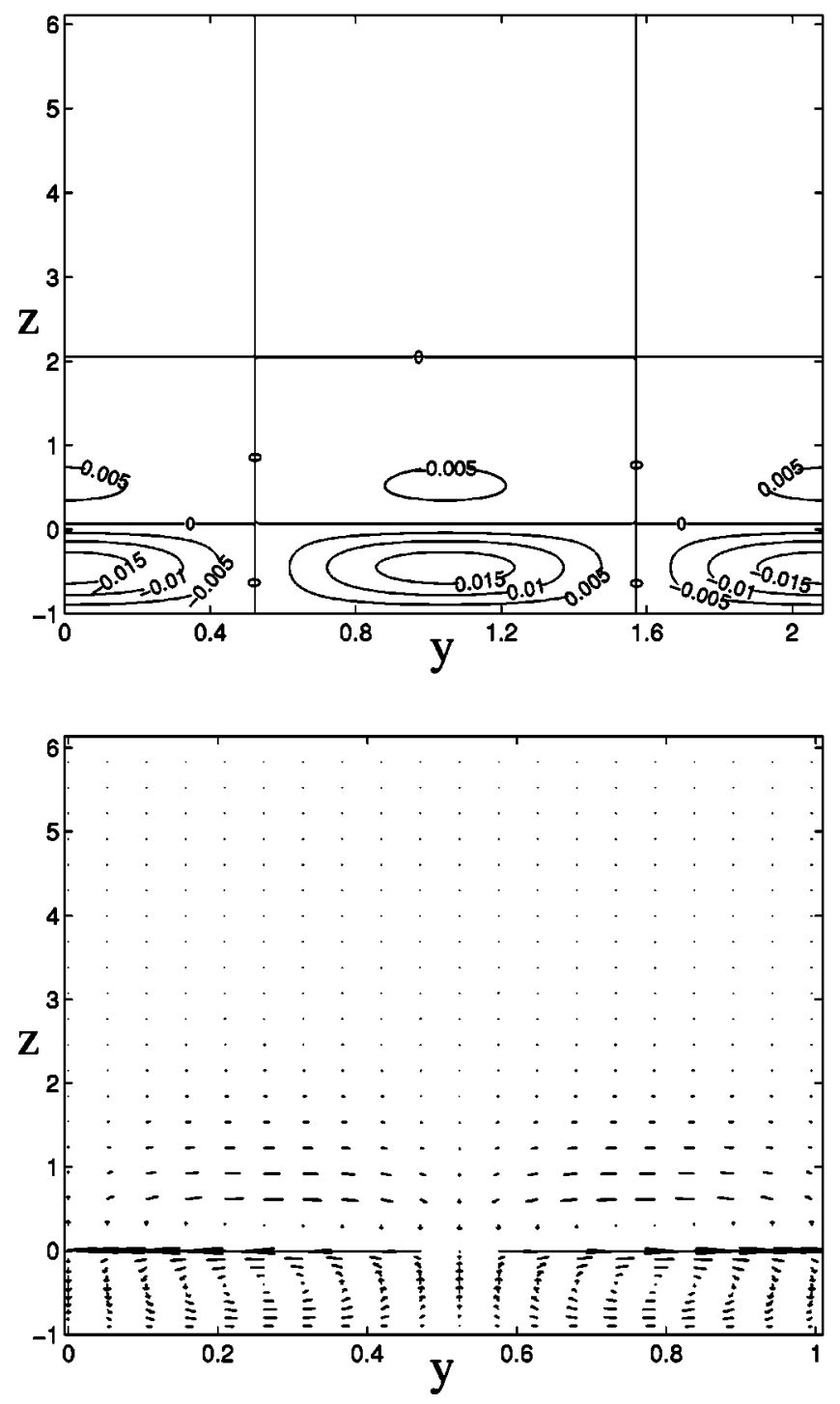

(b)
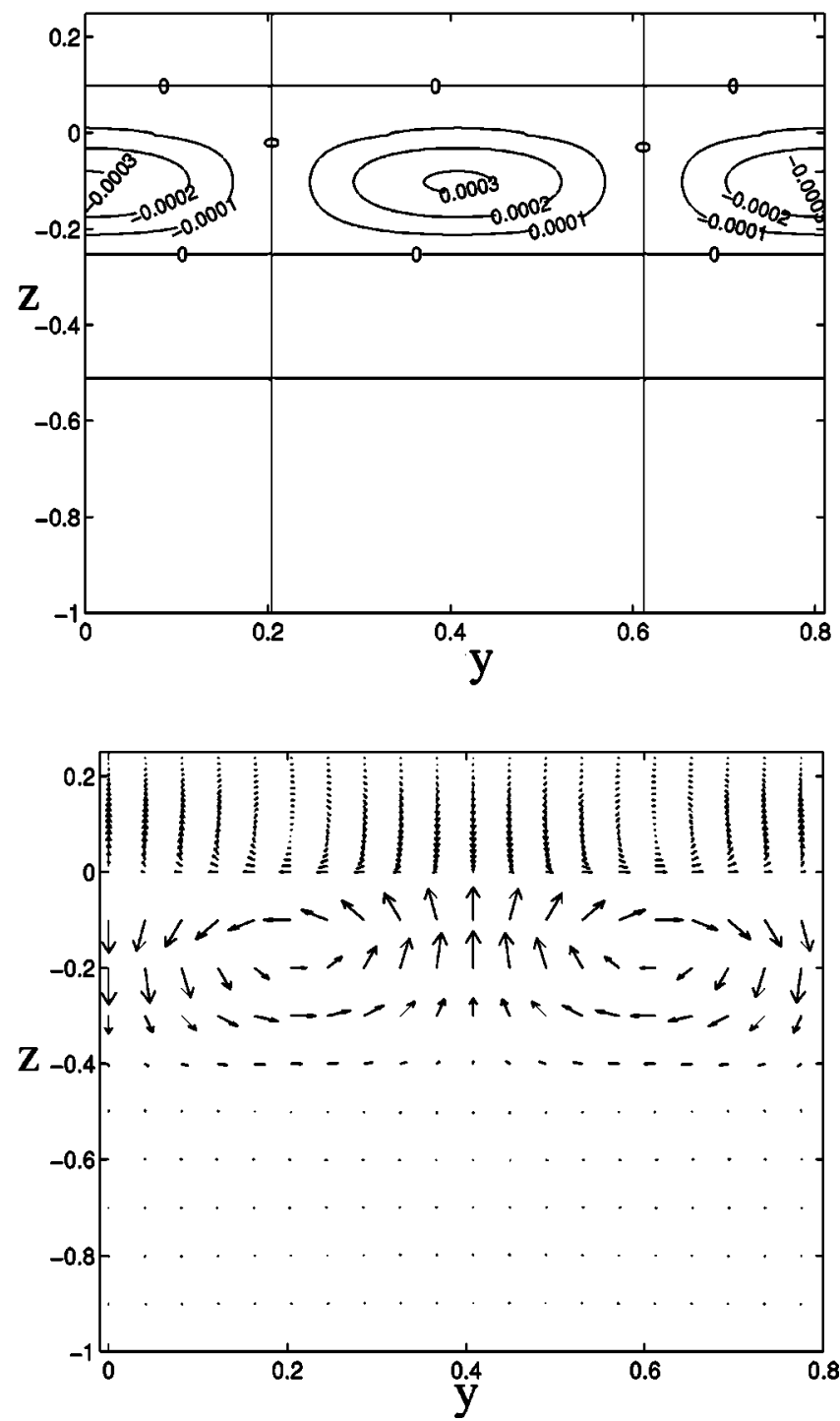

FIG. 5. Isotherms (above) and velocity fields (below) in the plane $z-y$ are represented at critical thresholds for $5 \mathrm{cS}$ silicon oil/HT-70 system and $h_{T}=9 \mathrm{~mm}$, for two bottom depth ratios corresponding to different branches: $\hat{h}_{1}=14 \%$ (right) and $\hat{h}_{1}=80 \%$ (left).

$z=0$, where they are less important. A similar behavior is exhibited by the velocity fields. For large fractional bottom depths $\left[\hat{h}_{1}=80 \%\right.$ in Fig. 5 (b) $]$ located at the other branch the temperature gradients are concentrated in liquid 1 near the interface and do not penetrate into the bulk of the lower liquid, and the velocity field is the largest in the lower liquid and is felt roughly at the same depth than the temperature gradients. Other stationary branches exhibit different kind of behaviors.

\section{B. Three-dimensional perturbations}

However, one cannot exclude the possibility that the most unstable disturbances are three dimensional. It follows that a minimization process with respect to $k_{x}$ and $k_{y}$ is required to find the critical parameters. A typical stability surface is shown in Fig. 6. In the presence of lateral heating the reflec-

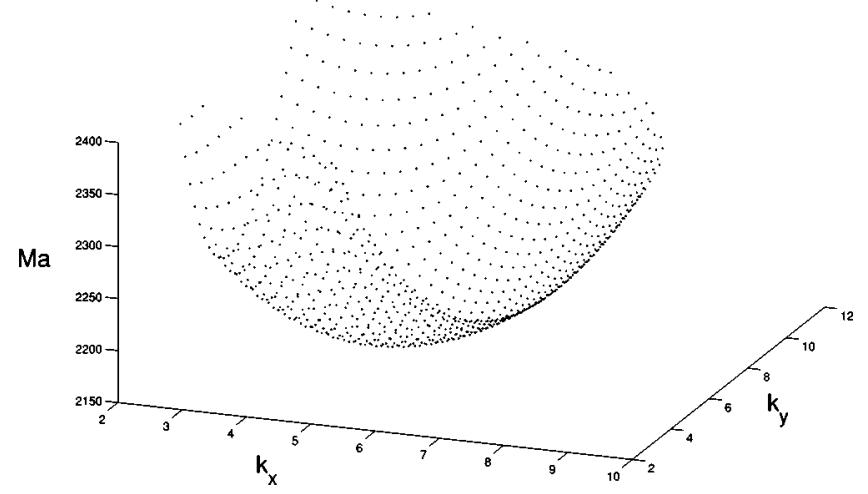

FIG. 6. Surface of neutral stability for three-dimensional perturbations. In this example $h_{T}=6 \mathrm{~mm}$, and $\hat{h}_{1}=78 \%$. The breaking of $k_{x}, k_{y}$ symmetry is clearly appreciated. The stability threshold is located at $\mathbf{k}=(4.2,7)$. 

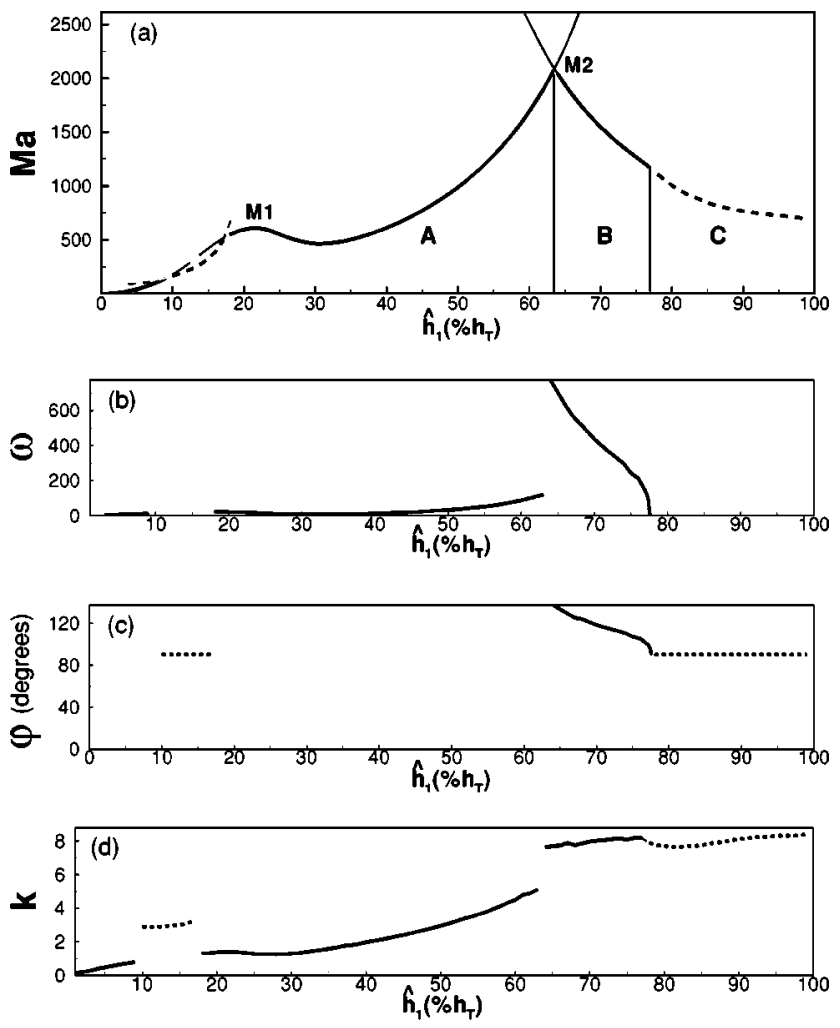

FIG. 7. Critical parameters for the system $5 \mathrm{cS}$ silicon oil (upper liquid) and HT-70 (lower liquid) with total depth $9 \mathrm{~mm}$. (a) Critical Marangoni, (b) critical frequency, (c) angle of the critical wave vector, and (d) modulus of the critical wave vector. Solid line: oscillatory behavior, dashed line: stationary behavior. In the points where there is an interaction between two branches, their extensions in the Marangoni curve are displayed. Different characteristic patterns are obtained in regions $A, B$, and $C$ (see the text).

tion symmetry in the direction $x$ is broken. Hence the marginal surface is only symmetrical under the changes $k_{y} \rightarrow$ $-k_{y}$. The marginal surface is, in general, more complicated because several intermingled branches arise. The position of the minimum is given by the $k_{x}$ and $k_{y}$ components of the critical wave number, which in the case of oscillatory instability also determine the direction of propagation $\varphi$ of the waves. Recalling that the positive $x$ axis is directed from the cold to hot side according to Eq. (7), the hydrothermal waves will propagate from cold to hot for $\varphi \in\left[0^{\circ}, 90^{\circ}\right]$, in contrast for $\varphi \in\left[90^{\circ}, 180^{\circ}\right]$ the direction of propagation is the opposite. Note that the symmetry $(y \rightarrow-y)$ of the linear problem makes that the waves with wave number $\left(k_{x}, \pm k_{y}\right)$ become unstable simultaneously. From now on, we will restrict to $\varphi \in\left[0^{\circ}, 180^{\circ}\right]$ without the loss of generality.

In Fig. 7 are represented the critical Marangoni number, frequency, angle, and modulus of the wave number for the system HT-70 (lower liquid) and $5 \mathrm{cS}$ silicon oil (upper liquid) with $h_{T}=9 \mathrm{~mm}$. The critical Marangoni curve exhibits a local maximum $M 1$ and a local minimum at $\hat{h}_{1}=31 \%$. The Marangoni number reaches its maximum value in $M 2$, which is a crossing point between two branches.
TABLE II. Position of $M 2$ as a function of the viscosity ratio (left) and thermal viscosity ratio (right) of the two liquids. The case $\nu=1, \kappa=1$ corresponds to an ideal configuration formed by two layers of the same fluid ( $5 \mathrm{cS}$ silicon oil).

\begin{tabular}{lccc}
\hline \hline$\nu$ & $\hat{h}_{1}(\%)$ & $\kappa$ & $\hat{h}_{1}(\%)$ \\
\hline 0.1 & 84 & 0.1 & 90 \\
1 & 81 & 1 & 81 \\
10 & 68 & 10 & 62 \\
\hline \hline
\end{tabular}

Beyond $M 2$, the behavior of the critical Marangoni curve is the same as in a one-layer system filled by the lower fluid. In such a case the critical Marangoni number also decreases and the spanwise wave number increases with the depth [5].

The position of $M 2$ depends on the physical properties of both fluids. To study the effect of the transport coefficients, i.e., viscosity $\nu$ and thermal diffusivity $\kappa$ on the position of $M 2$, we have considered an ideal system formed by two layers of $5 \mathrm{cS}$ silicon oil, which differ only by their values of $\nu$ and $\kappa$. In Table II are given the positions of $M 2$ for different values of the transport coefficients. By increasing the thermal diffusivity and viscosity of the lower liquid, the position of $M 2$ is shifted towards higher values of $\hat{h}_{1}$; on the contrary, if $\nu$ and $\kappa$ are increased in the upper liquid the shift is towards lower values of $\hat{h}_{1}$. The location of $M 2$ depends also on the geometry of the system: an increase of $h_{T}$ produces a shift towards a greater $\hat{h}_{1}$, however, for high $h_{T}$ values the shift becomes saturated, as shown by the Marangoni curves of Figs. 7 and 8.

Examination of the vertical profile of temperature of the basic state allows us to determine the positions of the local maximum $M 1$ at $21 \%$ and of the local minimum at about $31 \%$. Figure 3(b) displays the vertical profile of temperature; it is seen that, for $h_{T}=9 \mathrm{~mm}$, starting from the region (I) and decreasing $\hat{h}_{1}$ up to $31 \%$ the unstable vertical profile of the upper fluid splits in two smaller unstable sublayers and a third one in the middle, which is stable. The shape of the temperature profile remains unchanged in liquid 1.

In the configuration represented by region (III), there is a greater extension of the stable regions than in (I), thus the scenario in (III) consists in an increase of Ma due to the progressive stabilization of the upper layer when $\hat{h}_{1}$ decreases. The border between regions (I) and (III) gives the position of the local minimum of Fig. 7(a). However, when region (IV) is attained, the stratification in liquid 1 becomes unstable and the whole system is more and more unstable, with a decrease of Ma. This finds its roots in the two driving forces (thermocapillary and buoyancy), which are both destabilizing and cooperatives for the perturbations undergone by the lower liquid. As a consequence, the border between regions (III) and (IV) of Fig. 3(b) gives the position of $M 1$. Inside (IV) the decrease of Ma when $\hat{h}_{1}$ is lowered reflects the greater importance of the destabilizing effect of the interfacial tension. The position of $M 1$ is predicted by the vertical temperature profile of the basic state and it will not be influenced by the Prandtl number. 
(a)
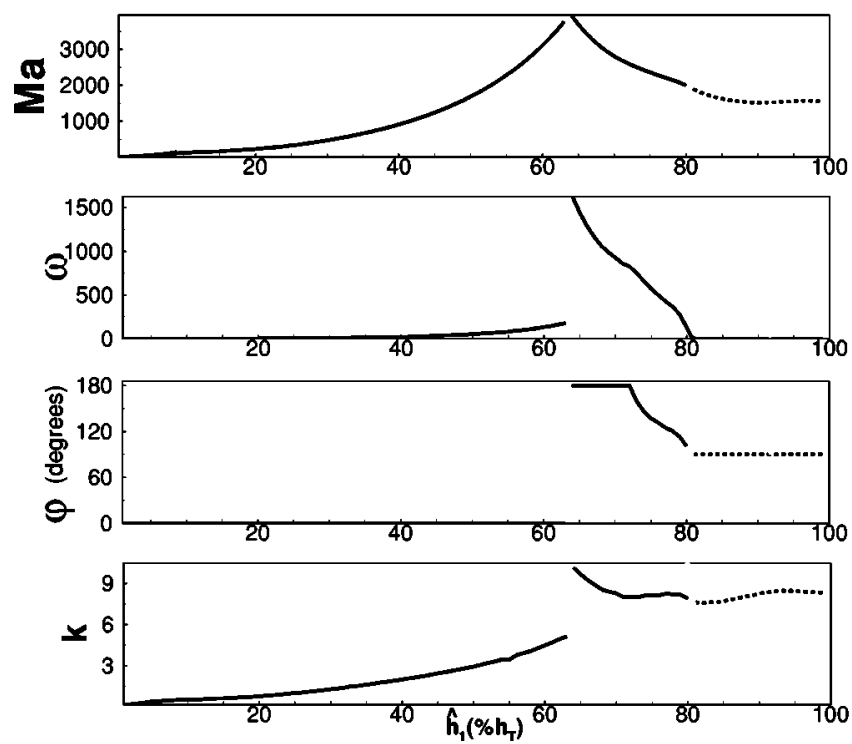

(b)
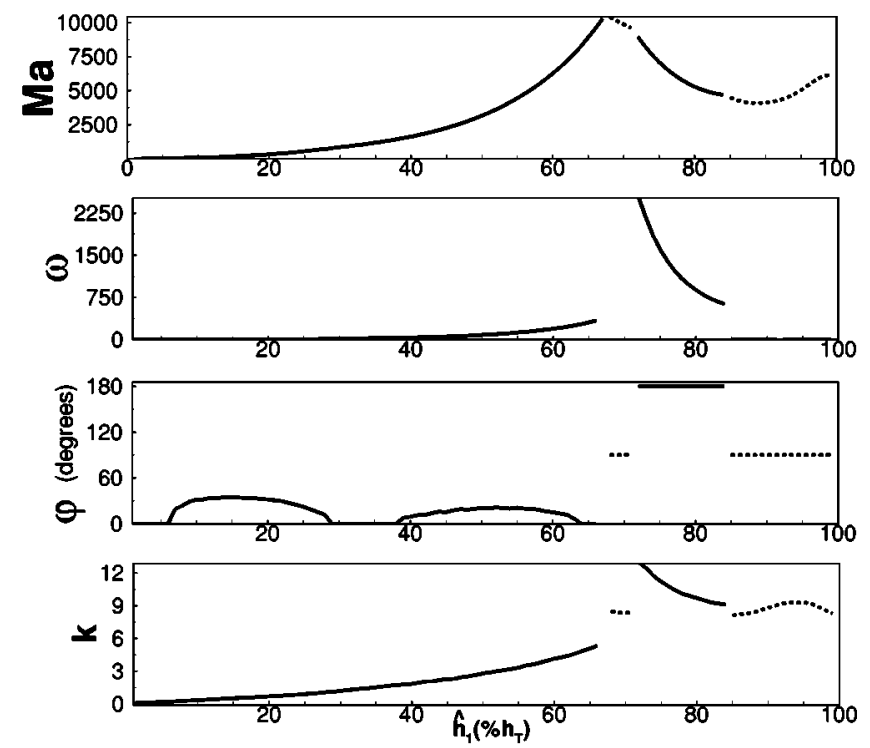

FIG. 8. Critical parameters for the system HT-70 (lower liquid) and $5 \mathrm{cS}$ silicon oil (upper liquid) with total depths $6 \mathrm{~mm}$ (a) and $3 \mathrm{~mm}$ (b) Solid line: oscillatory behavior, dashed line: stationary behavior.

One distinguishes three main regions $A, B$, and $C$, in the curves of Figs. 7 and 8 giving the critical parameters as a function of the relative depth of the liquids.

\section{Region A}

The first region $A$ extends from the lowest value of $\hat{h}_{1}$ until $M 2$. The critical Marangoni number increases with the drop of the depth of the upper liquid because of the decrease of the buoyancy effects in it, indicating that the upper liquid dominates the dynamics in $A$. The pattern in this region consists of hydrothermal waves with zero or small angles of propagation. They propagate from cold to hot regions with an angle parallel to the gradient of temperature for $h_{T}=6$ and $9 \mathrm{~mm}$; deviating to greater angles when the thermocapillary effects are relatively more important, as for $h_{T}$ $=3 \mathrm{~mm}$. Concerning the oscillatory modes, the angle of propagation is similar to single liquids with a high $\operatorname{Pr}[3]$. As seen in Fig. 7(d), region $A$ is characterized by an increase of $|\mathbf{k}|$ with $\hat{h}_{1}$, with a small dependence of $|\mathbf{k}|$ on $h_{T}$. At $h_{T}$ $=9 \mathrm{~mm}$, where the buoyancy effects are more important, one finds a range of depth ratios $6 \%<\hat{h}_{1}<16 \%$ where the critical modes take the form of stationary rolls. At the borders of this window, there is a codimension-two (cod-2) point formed by the interaction between a Hopf mode and a stationary mode of different wave numbers. Such cod-2 points were also found in two-liquid layers heated from below [24].

\section{Region B}

Region $B$ is characterized by a strong jump of the frequency at $M 2$ for $h_{T}=6$ and $9 \mathrm{~mm}$, and weakly shifted by $\Delta \hat{h}_{1}=4$ for $h_{T}=3 \mathrm{~mm}$. For $h_{T}=6$ and $9 \mathrm{~mm}, M 2$ is characterized by a pair of Hopf modes with finite and different wave numbers and frequencies which are simultaneously critical. At this point of cod-2 the configuration is the most stable with the greatest Ma. The difference between the wave numbers of the two Hopf modes is due to the occurrence of a large spanwise component for $h_{T}=9 \mathrm{~mm}$, while for $h_{T}$ $=6 \mathrm{~mm}$ there exists only a jump in the streamwise component. To our knowledge, this kind of interaction between two oscillatory modes has not yet received attention in BénardMarangoni problems. For $h_{T}=3 \mathrm{~mm}$, the presence of a small stationary branch near M2 prevents the existence of this kind of interaction, and the cod- 2 point is generated by a stationary mode and a Hopf mode. Concerning the hydrothermal waves, the corresponding angles of propagation are greater than the angles of propagation in region $A$. The direction of propagation of the hydrothermal waves in this region is reversed, propagating from the hot to the cold side. The width of region $B$ decreases with $h_{T}$, from $\Delta \hat{h}_{1}=18 \%$ for $h_{T}=9 \mathrm{~mm}$ to $\Delta \hat{h}_{1}=14 \%$ for $h_{T}=3 \mathrm{~mm}$. The modulus of the wave number varies in a more complicated way than in the region $A$, and is more sensitive to the total depth. In region $B$, the strong competition between the two layers is responsible for the oscillatory convection.

\section{Region $C$}

The transition from the second region $B$ to the third region $C$ is smooth with a monotonous decrease of the frequency of the oscillatory branch. The third region is characterized by stationary longitudinal rolls and extends up to the highest $\hat{h}_{1}$. It spreads towards lower $\hat{h}_{1}$ when the total depth is increased. The dynamics of this region is dominated by the lower layer. The qualitative behavior of the modulus $k$ of the wave number is practically unaffected by the overall depth.

In Fig. 9(a) are shown the critical parameters for the bilayer formed by perfluorinated hydrocarbon Fc-75 (lower liquid) and water (upper fluid), whereas in Fig. 9(b) are reproduced the results for the couple acetonitrile (lower liquid) and $n$-hexane (upper liquid). Both setups have been experi- 
(a)
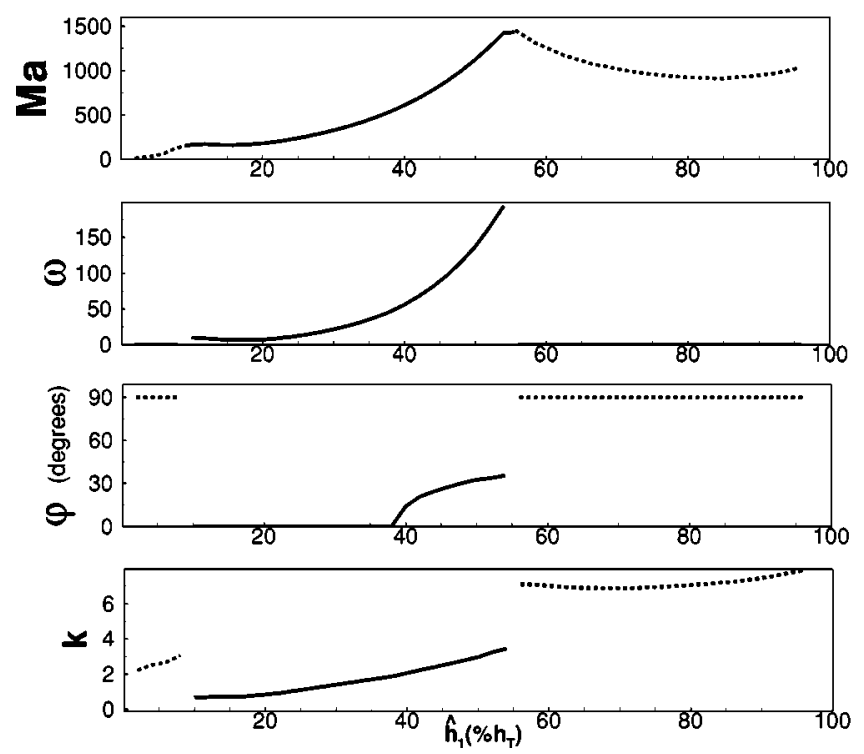

(b)
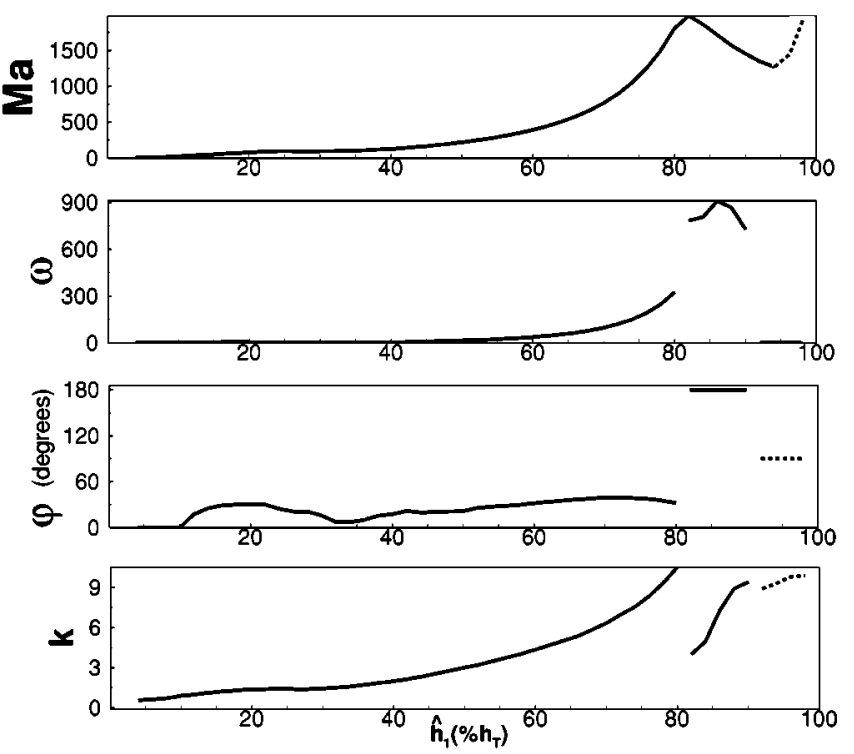

FIG. 9. Critical parameters for the pair Fc-75 (lower liquid) and water (upper liquid) (a) and for the pair acetonitrile (lower liquid) and $n$-hexane (upper liquid) (b). The total depth for both configurations is $h_{T}=6 \mathrm{~mm}$.

mentally studied in the case of a vertical heating [13-15]. The instability thresholds are evaluated for a total depth of 6 $\mathrm{mm}$ in both configurations. The critical Marangoni curves exhibit maxima at $M 1$ and $M 2$ as discussed above. In the case Fc-75/water, hydrothermal waves which propagate from cold to hot in the region located between $M 1$ and $M 2$ are found, for the other values of the fractional bottom depth stationary longitudinal rolls are forecast. The system acetonitrile/n-hexane presents the same three regions as exhibited by the pair $5 \mathrm{cS}$ silicon oil/HT-70 with a cod-2 point created by two oscillatory modes with different frequencies and wave numbers. The general features discussed earlier in this section remain valid for all the cases which have been examined.

Tuning the relative depths of both layers, according to our theoretical study it would be possible to obtain in the same experimental configuration with a fixed pair of liquids all the patterns observed in different single-layer systems: from longitudinal stationary rolls to hydrothermal waves with directions of propagation ranging from small angles of propagation (as in one-liquid systems with high Pr) to high angles (as in one-liquid systems with small Pr).

\section{CONCLUSIONS}

We have investigated the onset of convection in systems constituted by horizontal liquid layers subjected to a lateral heating. The system is bounded by two rigid and thermally conductive horizontal walls and the interface is supposed to be nondeformable. The acting forces are the interfacial tension and the gravity. They are cooperative in the lower layer and competitive in the upper one. The number of parameters is greatly enhanced with respect to the case of one-layer problems. We have considered three different combinations of liquids, namely, bilayers of perfluorinated HT-70 and $5 \mathrm{cS}$ silicon oil, perfluorinated Fc-75 and water, and acetonitrile and $n$-hexane; these fluids have been considered because they have already been the subject of experiments involving a vertical heating [15]. It is our hope that the results of the present paper will motivate researchers to repeat these experiments with a lateral heating.

As soon as a horizontal temperature gradient is applied, a global circulation takes place in each layer. The temperature and velocity fields of the basic state have been derived and discussed. For a given experimental setup and a fixed heating, the two control parameters are the total depth and the depth ratio between the liquids. In a diagram representing the depth ratio versus the total depth, four regions for the temperature and velocity profiles have been distinguished. The main information drawn from this diagram can be summarized as follows. The interaction between interfacial tension and buoyancy can give rise to (1) a counterrotating cell in each layer, (2) two counterrotating cells in the upper liquid and one cell in the lower one, in both cases one observes a flow from hot to cold near the interface (3) two counterrotating cells in the lower liquid and a cell in the upper one; and (4) a counter-rotating cell in each liquid, but with the flow being dragged near the interface from cold to hot in the last two cases. We have also determined the different temperature profiles that result from the interplay between thermal diffusivity and fluid motion.

The linearized evolution equations for the perturbations of the basic state have been established and the ensuing eigenvalue problem has been solved. Bidimensional and threedimensional perturbations have been respectively considered. A physical interpretation of the different regions found has been proposed. The interest of the present analysis with respect to a one-layer system is that it exhibits a great variety of behaviors depending on the nature of the liquids, the total depth, and the relative depth between the layers. Among the 
most interesting results can be mentioned the occurrence of a cod-2 point arising as a consequence of the interaction between two Hopf modes with different frequencies and wave numbers. Furthermore, three different patterns are predicted: (1) hydrothermal waves propagating from cold to hot side with a small angle, which is typical of lateral heating in one-liquid systems with high Pr, (2) hydrothermal waves propagating from hot to cold, and (3) longitudinal stationary rolls.

A variety of results confirms that the problem of lateral heating in two-layer systems is a promising and interesting area of research, both from the theoretical and experimental points of view. It would be specially interesting to study the system close to $M 2$ when we interplay between two oscillatory modes.

\section{ACKNOWLEDGMENTS}

The authors wish to thank Mireille Dondlinger, Thomas Desaive (Liege), and Jean Bragard (Pamplona) for useful discussions. This work has been supported by the European Union through Contract No. ICOPAC HPRN-CT-200000136, by the DGICYT (Spanish Government) Grant No. BFM2002-0201, and the PIUNA (Universidad de Navarra). One of us (S.M.) also acknowledges the financial support from the "Asociación de Amigos de la Universidad de Navarra."

\section{APPENDIX}

Expressions of the velocity and temperature profiles of the basic state in dimensionless units:

$$
\begin{aligned}
u_{0}^{(1)}= & \frac{1}{48(a+\mu) \nu}\left[-12 a \operatorname{Ma} \nu\left(1+4 z+3 z^{2}\right)+R a\left\{a^{3} \alpha \mu\right.\right. \\
& -a \nu+4 \mu\left(a^{3} \alpha+\nu\right) z+3\left(a^{3} \alpha \mu+3 a \nu+4 \mu \nu\right) z^{2} \\
& \left.\left.+8(a+\mu) \nu z^{3}\right\}\right],
\end{aligned}
$$

$$
\begin{aligned}
u_{0}^{(2)}= & \frac{1}{48 a(a+\mu) \nu}\left[-12 \operatorname{Ma} \nu\left(a^{2}-4 a z+3 z^{2}\right)+\operatorname{Ra}\left\{a^{4} \alpha \mu\right.\right. \\
& -a^{2} \nu+4 a\left(a^{3} \alpha+\nu\right) z-3\left(4 a^{3} \alpha+3 a^{2} \alpha \mu+\nu\right) z^{2} \\
& \left.\left.+8 a \alpha(a+\mu) z^{3}\right\}\right],
\end{aligned}
$$

$$
\begin{aligned}
\tau^{(1)}= & \frac{1}{2880(a+\lambda)(a+\mu) \nu \kappa}\left[6 0 \mathrm { Ma } \left\{a^{2} \nu(a \lambda+\kappa)+a \lambda \nu\left(a^{2}\right.\right.\right. \\
& -\kappa) z-6 a(a+\lambda) \nu \kappa z^{2}-8 a(a+\lambda) \nu \kappa z^{3}-3 a(a \\
& \left.+\lambda) \nu \kappa z^{4}\right\}+\operatorname{Ra}\left\{a \nu\left(5 a^{2} \lambda+9 a \kappa+4 \mu \kappa\right)-a^{4} \alpha\left(4 a^{2} \lambda\right.\right. \\
& +9 a \lambda \mu+5 \mu \kappa)-\left[\nu\left(-5 a^{3}+9 a \kappa+4 \mu \kappa\right)+\alpha\left(4 a^{6}\right.\right. \\
& \left.\left.+9 a^{5} \mu-5 a^{3} \mu \kappa\right)\right] \lambda z+30 a(a+\lambda)\left(a^{2} \alpha \mu-\nu\right) \kappa z^{2} \\
& +40(a+\lambda) \mu\left(a^{3} \alpha+\nu\right) \kappa z^{3}+15(a+\lambda)\left(a^{3} \alpha \mu+3 a \nu\right. \\
& \left.\left.+4 \mu \nu) \kappa z^{4}+24(a+\lambda)(a+\mu) \nu \kappa z^{5}\right\}\right],
\end{aligned}
$$

$$
\begin{aligned}
\tau^{(2)}= & \frac{-1}{2880 a(a+\lambda)(a+\mu) \nu \kappa}\left[6 0 \mathrm { Ma } \left\{-a^{3} \nu(a \lambda+\kappa)+a^{2} \nu\right.\right. \\
& \left(-a^{2}+\kappa\right) z+6 a^{2}(a+\lambda) \nu z^{2}-8 a(a+\lambda) \nu z^{3} \\
& \left.+3(a+\lambda) \nu z^{4}\right\}+\operatorname{Ra}\left\{a ^ { 2 } \left[-\nu\left(5 a^{2} \lambda+9 a \kappa+4 \mu \kappa\right)\right.\right. \\
& \left.+a^{3} \alpha\left(4 a^{2} \lambda+9 a \lambda \mu+5 \mu \kappa\right)\right]+a\left[\nu \left(-5 a^{3}+9 a \kappa\right.\right. \\
& \left.+4 \mu \kappa)+\alpha\left(4 a^{6}+9 a^{5} \mu-5 a^{3} \mu \kappa\right)\right] z-30 a^{2}(a+\lambda) \\
& \times\left(a^{2} \alpha \mu-\nu\right) z^{2}-40 a(a+\lambda)\left(a^{3} \alpha+\nu\right) z^{3}+15(a+\lambda) \\
& \left.\left.\times\left[a^{2} \alpha(4 a+3 \mu)+\nu\right] z^{4}-24 a \alpha(a+\lambda)(a+\mu) z^{5}\right\}\right] .
\end{aligned}
$$

Note that the lower fluid (superscript 1) is defined in the interval $z \in[-1,0]$ and the upper fluid (superscript 2) in the interval $z \in[0, a]$, according to the definitions given in Sec. II.
[1] M.F. Schatz and G.P. Neitzel, Annu. Rev. Fluid Mech. 33, 93 (2001).

[2] M.K. Smith and S.H. Davis, J. Fluid Mech. 132, 119 (1983).

[3] M.K. Smith, Phys. Fluids 29, 3182 (1986).

[4] P. Parmentier, V. Regnier, and G. Lebon, Int. J. Heat Mass Transfer 36, 2417 (1993).

[5] G.Z. Gershuni, P. Laure, V.M. Myznikov, B. Roux, and E.M. Zhukhovitsky, Microgravity Q. 2, 141 (1992).

[6] J.F. Mercier and C. Normand, Phys. Fluids 8, 1433 (1996).

[7] F. Daviaud and J.M. Vince, Phys. Rev. E 48, 4432 (1993).

[8] J. Priede and G. Gerbeth, Phys. Rev. E 56, 4187 (1997).

[9] R.J. Riley and G.P. Neitzel, J. Fluid Mech. 359, 143 (1998).

[10] M.K. Smith, J. Fluid Mech. 194, 391 (1988).

[11] M.A. Pelacho, A. Garcimartín, and J. Burguete, Phys. Rev. E 62, 477 (2000); J. Burguete, N. Mukolobwiez, F. Daviaud, N. Garnier, and A. Chiffaudel, Phys. Fluids 13, 2773 (2001).
[12] W.A. Tokaruk, T.C.A. Molteno, and S.W. Morris, Phys. Rev. Lett. 84, 3590 (2000).

[13] D.D. Joseph and Y.Y. Renardy, Fundamentals of Two-fluid Dynamics (Springer, Berlin 1993); I.B. Simanovski and A.A. Nepomnyashchy, Convective Instabilities in Systems with Interface (Gordon and Breach, London, 1993); P. Colinet, P. Géoris, J.C. Legros, and G. Lebon, Phys. Rev. E 54, 514 (1996); A. Engel and J.B. Swift, ibid. 62, 6540 (2000).

[14] C.D. Andereck, P.W. Colovas, M.M. Degen, and Y.Y. Renardy, Int. J. Eng. Sci. 36, 1451 (1998).

[15] A. Juel, J.M. Burgess, W.D. McCormick, J.B. Swift, and H.L. Swinney, Physica D 143, 169 (2000).

[16] P. Cardin and H.C. Nataf, Europhys. Lett. 14, 655 (1991).

[17] D. Villers and J.K. Platten, Appl. Sci. Res. 45, 145 (1988); 47, 177 (1990).

[18] T. Doi and J.N. Koster, Phys. Fluids A 5, 1914 (1993). 
[19] Q.S. Liu, G. Chen, and G. Roux, Int. J. Heat Mass Transfer 36, 101 (1993).

[20] Q.S. Liu, B. Roux, and M.G. Velarde, Int. J. Heat Mass Transfer 41, 1499 (1998).

[21] J.K. Platten and D. Villers, in Physicochemical Hydrodynamics: Interfacial Phenomena, Vol. 174 of NATO Advanced Stud- ies Institute, Series B, Physics, edited by M.G. Velarde (Plenum, New York, 1988), p. 311.

[22] G. Carneiro and C. Pérez-García, Phys. Fluids 3, 292 (1991).

[23] V.C. Regnier, P.C. Dauby, and G. Lebon, Phys. Fluids 12, 2787 (2000).

[24] K. Fujimura and Y. Renardy, Physica D 85, 25 (1995). 